\title{
Higher-order Brunnian structures and possible physical realizations
}

\author{
N. A. Baas \\ Department of Mathematical Sciences, NTNU, NO-7491 Trondheim, Norway
}

\author{
D. V. Fedorov, A. S. Jensen, K. Riisager, A. G. Volosniev, and N. T. Zinner \\ Department of Physics and Astronomy, Aarhus University, DK-8000 Aarhus C, Denmark
}

\begin{abstract}
We consider few-body bound state systems and provide precise definitions of Borromean and Brunnian systems. The initial concepts are more than a hundred years old and originated in mathematical knot-theory as purely geometric considerations. About thirty years ago they were generalized and applied to the binding of systems in nature. It now appears that recent generalization to higher order Brunnian structures may potentially be realized as laboratory made or naturally occurring systems. With the binding energy as measure, we discuss possibilities of physical realization in nuclei, cold atoms, and condensed matter systems. Appearance is not excluded. However, both the form and the strengths of the interactions must be rather special. The most promising subfields for present searches would be in cold atoms because of external control of effective interactions, or perhaps in condensed-matter systems with non-local interactions. In nuclei, it would only be by sheer luck due to a lack of tunability.
\end{abstract}

\section{MOTIVATION}

Tying knots has from ancient times been a very practical skill to master. Two circular closed strings (rings) can be tied together and only removed from each other after cutting one of them. Such arrangements are called linked geometries. With more than two rings numerous possibilities arise for distinctly different topological structures. If one part cannot be removed from another part without untying or cutting, these parts are called linked and if removable called unlinked. The mathematical classification and description of such geometric or topological structures are called knot theory, and it has a hundred year long history.

One of the simplest cases is obviously three rings. They can be arranged so that they are linked, but if any one is cut and removed the two remaining ones can be removed without further cutting. This is illustrated in figure 1. This construction is now often called Borromean rings, since they are the heraldic symbol of the dukes of Borromeo. However, the structure was fascinating already in the Norse mythology, shown as triangles and known as Odins knot, and in various disguises especially in religious contexts throughout history. Not surprisingly psychology (emphasized by for instance Jacques Lacan) could use it a ambly as symbols of interlocked triads like past present and future, etc.

In science, the Borromean rings have triggered several generalizations. The present paper discusses whether a new family of generalized structures may have counterparts in physics. We shall start by giving a brief review of previous work. Several early papers [1, 2] preceeded the first mathematically rigorous treatments [3, 4] in the 1960's. Since then links of multiplicity $n$, essentially $n$ (deformed) rings in three-dimensional space, are called Brunnian if they form a connected structure where all sybsystems are unlinked.

A link is of type $B(n, k)$ if it consists of $n$ rings in such

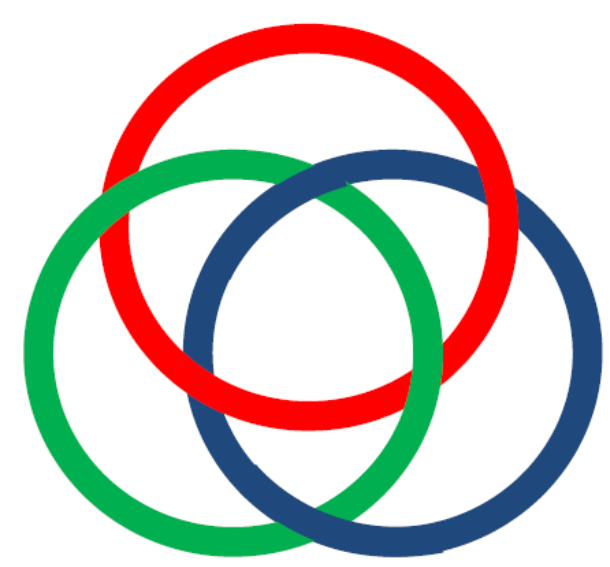

FIG. 1: Illustration of the Borromean rings which are held together only by the presence of all three. If any one of the rings is cut open, the entire structure falls apart.

a way that any subsystem of $m$ rings with $m \leq k$, is unlinked. For $k=2$ the link is called Borromean, for $k=n-1$ it is called Brunnian. If $n=3$ the two notions coincide. The notion of $B(n, k)$ links was introduced in [5, 6] extending previous notions in 7].

Further generalizations of higher order links - in particular higher order Brunnian links were made in [5] as special case of higher order structures introduced in [8], expanded in [9]. First order structures are for example Brunnian links, second order structures are Brunnian rings of Brunnian rings, etc., see figures [13. See [5] for more illustrations of higher order links.

We shall focus on the occurence on related higher-order structures in physics, but should point out first that more direct examples may be found in chemistry or biology. Molecular rings with non-standard topologies have been contemplated for a long time [10] and may in certain cases 
have different properties (such as optical activity). Biological molecules are known to form long chains of folded structures, often with knots. Borromean rings have been constructed from DNA [11] and even high-order topological structures have been envisaged [6].

In [5] it was proposed that higher order links for example of Brunnian type may suggest new physical states. The concept of Borromean systems was introduced in microscopic physics [12] based not on geometrical linking (topology), but on binding energy. We shall in a similar manner take over all of the above topological concepts by replacing geometrically (un)linked by energetically (un)bound. Note that since the exact geometrical configurations now are of less direct importance we can (and will) also consider two-dimensional structure rather than confining ourselves to three spatial dimensions. In the following section we shall give a general discussion of the possibilities for having higher order Brunnian systems. This is followed by more specific looks at atomic, condensed matter and nuclear systems.

\section{PHYSICAL REALIZATIONS OF HIGHER ORDER BRUNNIAN SYSTEMS}

In order to realize higher-order Brunnian systems in physical setups, one needs to consider multi-particle dynamics. For concreteness, we will focus here on the case of a second-order Brunnian state built from nine particles. At first we discuss the basic Hamiltonian setup and the interactions that one can expect. We then go on to consider subfields of physics where such a system might be accessible. This includes ultracold atomic gases of both non-polar and polar single- and two-species gases and nuclear systems. We also make some comments about potential relevance of higher-order Brunnian structures in traditional condensed-matter and solid-state systems.

Assume that we have three species of particles, $a, b, c$, which could be distinguished by either internal quantum numbers (f.x. electron spin or hyperfine spin in neutral atoms) or external quantum numbers (f.x. different mass particle). At this point we do not assume anything about the quantum statistics of the different species and they can as such be either fermionic, bosonic, or a mixture of the two. We assume that the system has two-body interaction terms between the same and different species, which we denote $V_{a a}, V_{b b}, V_{c c}, V_{a b}, V_{b c}, V_{a c}$ with the subscript indicating the particular pair of particles. These interactions are indicated on figure 3 .

In order to have a second-order Brunnian system of nine particles, we need to have three Borromean threebody systems, that is three bound three-body subsystems wherein no two-body interaction supports a bound state. This is well-known to occur on the weakly-interacting side of a Feshbach resonance in cold atomic gas systems that display the Efimov effect 13]. Numerically, such a state can be produced by using model potentials such

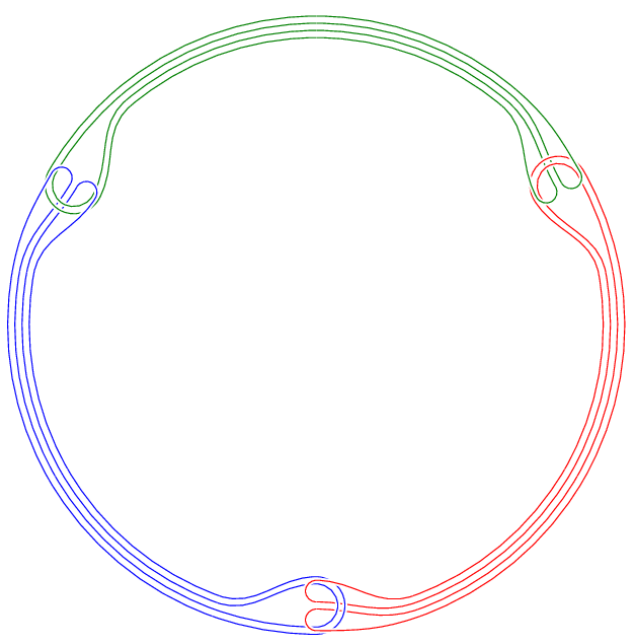

(a)A first order Brunnian ring

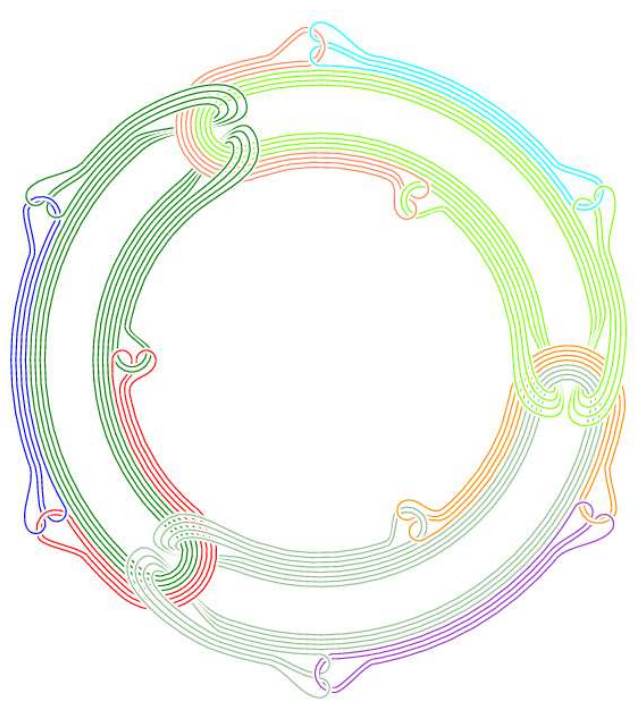

(b)A second order Brunnian ring

FIG. 2: Brunnian rings of first and second order

as Gaussian, or Yukawa type interactions with interaction ranges that are short compared to the overall size of the Borromean three-body states [14]. In this type of setup we are in a sense working effectively with three Borromean systems and ask when those three will form a bound structure (see figure 3). The idea is then that the effective interaction between these three-body Borromean clusters should support a Borromean state, i.e. if you remove one cluster you break the system into three unbound three-body states where each of the three-body systems form a Borromean state.

The total potential is

$$
V=V_{a a}+V_{b b}+V_{c c}+V_{a b}+V_{b c}+V_{a c}
$$

and the Schrödinger equation in turn becomes

$$
H \Psi:=(T+V) \Psi=E \Psi,
$$




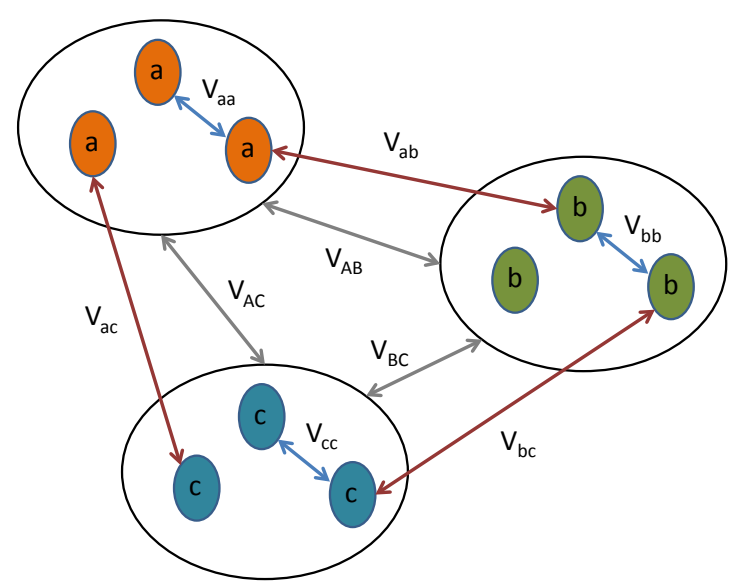

FIG. 3: A possible second-order Brunnian system with nine particles grouped into three different species, $a, b$, and $c$. The assumption is that each species holds a Borromean three-body bound state (indicated by the black rings). The intra-species interactions are denoted $V_{a a}, V_{b b}$, and $V_{c c}$, while the interspecies are $V_{a b}, V_{b c}$, and $V_{a c}$. In an effective description, we assume that we have three clusters that interact through effective interactions $V_{A B}, V_{B C}$, and $V_{A C}$.

where $\Psi$ is the total (nine particle) wave function, $E$ is the energy, and $T$ is the relative kinetic energy operator (excluding the center of mass part). Pursuing the idea above that one should be able to describe the system as a clusterized structure with three Borromean three-body systems, we now restructure the Hamiltonian, $H$. Following the schematics in figure 3, we denote the interactions between each of the clusters as $V_{A B}, V_{B C}$, and $V_{A C}$, which generally depend on the two-body interactions with the lower case subscripts, $V_{i j}$. For a well-developed cluster structure it would be a good approximation to decompose the total wave function as $\Psi=\phi_{A} \phi_{B} \phi_{C} \Phi_{A B C}$, where $\phi_{i}(i=A, B, C)$ satisfies

$$
\left(T_{i}+V_{i}\right) \phi_{i}=E_{i} \phi_{i},
$$

where $T_{i}$ is the relative kinetic energy operator of the corresponding three-body system, and $V_{A}=V_{a a}(12)+$ $V_{a a}(23)+V_{a a}(13)$ denotes the potential of all pairs within the three-body cluster containing species $a$, and likewise for $B$ and $C$. The 'relative' wave function between the three clusters, $\Phi_{A B C}$, satisfies the equation

$$
\left(T_{A B C}+V_{A B}+V_{B C}+V_{A C}\right) \Phi_{A B C}=E_{A B C} \Phi_{A B C},
$$

where

$T_{A B C}=-\frac{\hbar^{2}}{2 M_{A}} \vec{\nabla}_{\vec{R}_{C M}^{A}}-\frac{\hbar^{2}}{2 M_{B}} \vec{\nabla}_{\vec{R}_{C M}^{B}}-T_{C M}-\frac{\hbar^{2}}{2 M_{C}} \vec{\nabla}_{\vec{R}_{C M}^{C}}$,

is the kinetic energy operator of the relative motion of the clusters. Here we have introduced the total mass $M_{A}=3 m_{a}$ and the center-of-mass coordinate of the cluster $\vec{R}_{C M}^{A}$ (likewise for $B$ and $C$ ).
In order to achieve a realization of a second-order Brunnian system, one needs (i) to find a physical system where (4) supports a Borromean state, i.e. regime where no two-cluster subsystem is bound but the total three-cluster system is bound, and (ii) the subsystems governed by (3) must also be Borromean,

\section{ULTRACOLD ATOMIC GASES}

Advances in cold atomic gas physics [15, 16] implies that these systems now provide an effective simulation venue for testing various hypothesis of quantum mechanics, both for few- and many-body systems. In particular, the control over interactions is extremely precise through use of Feshbach resonances [17] and facilitates access to the strongly-coupled regime where typical perturbative approaches fail. Recently, cold polar molecules have been added to the toolbox [18, 19]. Heteronuclear molecules have a permanent dipole moment that is tunable and can provide a very strong long-range and anisotropic interaction. This extends the potential for simulation of quantum systems that are similar to materials since dipolar interactions can mimic the behavior of systems that have long-range Coulomb forces.

\section{A. Non-polar atomic gases}

First we consider the traditional case of non-polar atomic gases. These are typically made from singlespecies alkali atoms, but experiments have also been done with homonuclear molecules which, in absence of an external aligning field, will display no polar character. At the low densities and the low temperatures of experimentally realized atomic gases, the interactions that govern the dynamics of the systems have very short-range [16]. Since only low-energy scattering is allowed, this implies that the effective-range expansion can be used to describe the effects of interactions. With Feshbach resonances one can in fact tune the scattering length, $a$, to any desired value [17], and it is this feature that we will exploit.

In order to implement the three-species situation above we first consider bosonic alkali atoms and assume that we have populated three internal (hyperfine) states. This has been realized in experiments, effectively creating a spin-one system, i.e. bosons with three internal degrees of freedom [20, 21]. What is important is the particular scattering lengths in the different channels $a, b, c$. However, this requires that one can find a set of Feshbach resonances that will allow all intra-species scattering lengths to be tuned to the negative $a$ side, and close enough to the Feshbach resonance $(|a| \rightarrow \infty)$ where the Borromean bound state exists. At this point one could perhaps bind the second-order Brunnian nine particle system, perhaps with further tuning of the scattering lengths (without going to the $a>0$ side where two-body subsystem become bound in any of the three hyperfine components). 
A problem with the species one usually encounters for experiments with spin-one bosons like ${ }^{87} \mathrm{Rb}$, is that the background scattering length is positive, i.e. there is a two-body bound state between two atoms from the start. This means the system does not fulfil our aim without further fine-tuning.

The scenario with internal hyperfine state of a single atom is probably hard to achieve unless a fortunate set of resonances are present, or perhaps with suitable combinations of magnetic and optical resonances [17]. A different route would be to use different atoms as the different species. This requires use of inter-species Feshbach resonances instead. Again they should be bosonic atoms so as to allow a Borromean state to form in each cluster. Preferably, the intra-species interaction should have a negative background value of the scattering length so that no two-body bound state is present, in constrast to the ${ }^{87} \mathrm{Rb}$ system discussed above. Fortunately, there are some good alkali species available that have just this feature such as ${ }^{7} \mathrm{Li},{ }^{39} \mathrm{~K}$, or ${ }^{85} \mathrm{Rb}$. Thus far, however, interspecies interactions, resonances, and background parameters are largely unknown.

\section{B. Dipolar molecules}

We now proceed to discuss potential realization with polar molecules that have permanent dipole moments. In this case, externally applied AC and DC electric fields can be used to manipulate the interaction into various interesting regimes [22]. As discussed above, we are interested in a regime of Borromean binding. Thus we need to look for cases where two-body bound states are absent but three-body trimers can be bound nonetheless. However, the catch with dipolar molecules is that they can have very strong chemical losses due to strong head-to-tail attraction [18, 19]. This can be suppressed by confining the molecules in low-dimensional structures such as tubes or planes. This has been recently shown to reduce chemical losses significantly 23].

Interestingly, it is possible to create a two-dimensional setup and apply external fields to produce potentials that allow Borromean binding. In two dimensions, it is known that Borromean states can occur in potentials that have an inner attractive pocket and an outer repulsive tail 24]. Such a potential can in fact be achieved with polar molecules [22, 25]. A schematic depiction of this is shown in figure 4. Three identical fermionic particles interacting via such a potential will support a bound trimer when no two-body subsystem is bound [26].

Having fulfilled the first condition of presence of Borromean subsystems, we must now consider how to generate a nine particle three cluster system. However, with polar molecules we have long-range interactions. This implies that if we take a layered setup with three planes each containing one Borromean cluster, we could potentially achieve our goal of producing a second-order Brunnian structure. In figure 5 we show an illustration of

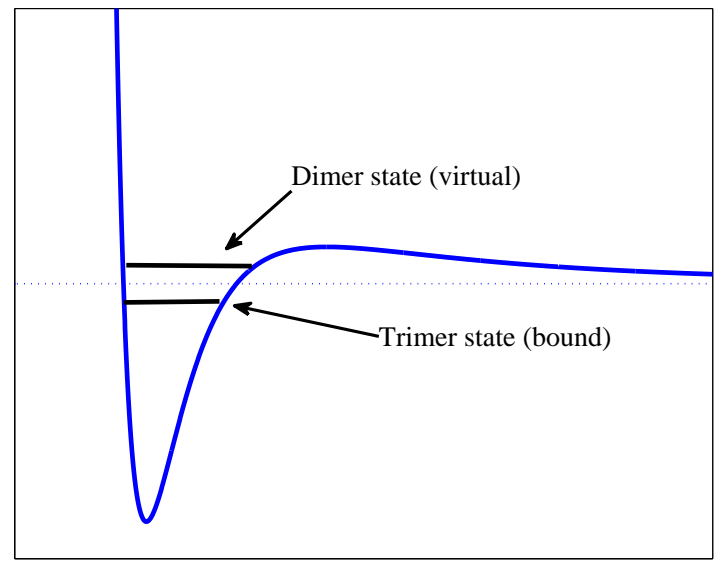

FIG. 4: Schematic illustration of a potential with an inner repulsive core, mid-range attractive pocket and an outer repulsive tail. The dotted horizontal line marks zero energy with respect to infinity where the potential vanishes. This potential shape can support a bound three-body trimer state even when the dimer state is unbound.

a trilayer setup. By adjusting the $\mathrm{AC}$ and $\mathrm{DC}$ external fields one tunes not only the intra-plane but also the inter-plane interactions, and it is not inconceivable that a regime can be found where the total structure of nine polar molecules is bound in a Borromean fashion such that removing one plane will break up the structure.

The DC field that is applied to the system will tend to align the molecules perpendicular to the layer planes as indicated by the arrows in figure 5. The interaction between particles in different layers holds a two-body bound state in the case of no AC field 27 29]. However, the presence of an $\mathrm{AC}$ field component will in general change the structure of the potential and can be used to push the two-body bound state above threshold 222]. We expect that the perfect conditions to look for a second-order Brunnian system would be somewhere around the threshold but with the two-body state slightly above (rendering it virtual).

In terms of experimental measurement, we imagine that one can use traditional RF spectroscopic techniques to probe the bound state structures [30]. The RF method works by driving transitions between different internal hyperfine states in the molecules. If one drives the molecules into a hyperfine state where the interaction potentials are very different and will not provide a bound structure, then the $\mathrm{RF}$ response pattern will display a structure at the binding energy of the initial state. This has been used very recently to detect Efimov states in association experiments [31, 32].

\section{CONDENSED-MATTER SYSTEMS}

In general many-body systems such as those found in condensed-matter and solid-state there are typically a hi- 


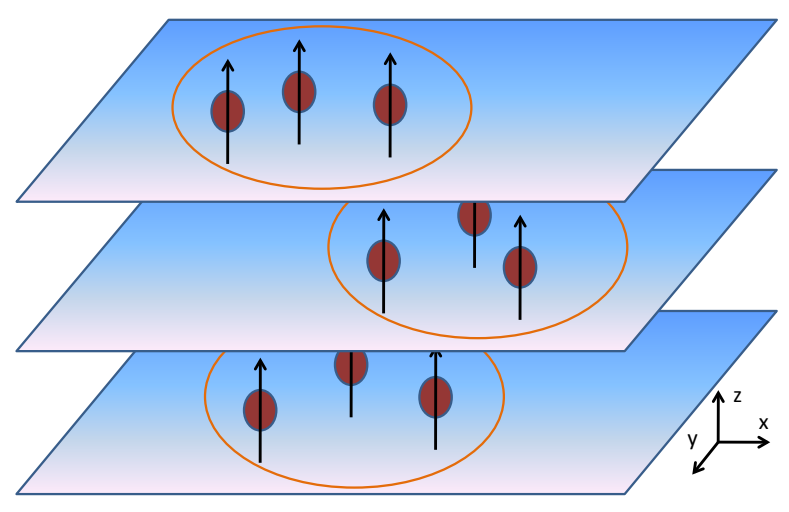

FIG. 5: A setup with three parallel planes containing polar molecules. An AC and DC electric field is applied to induce a specific potential between the dipoles in the planes and between the planes. The dipole moments are shown as arrows perpendicular to the planes. Through tuning of the potentials, one can achieve a situation where there are trimer bound states of three dipolar molecules in each plane. To achieve a second order Brunnian structure the total system across the planes would have to be bound such that removal of one trimer (or layer) makes the structure dissociate.

erachy of correlations. Depending on structure and density, the most important correlations tend to be those of relatively few particles. For instance, two-body correlations will be very pronounced in relation to weaklyinteracting Bose-Einstein condensates [33]. However, also three- and more-body correlations are important in such systems [34]. Recently, there has been a surge of activity into the regime of strong interactions, which can be achieved for instance by tuning parameters such that the scattering length diverges. Cold atoms have provided an experimental route to the study of this dynamics 35] which is considered a vital ingredient in understanding high-temperature superconducting materials [36]. This strongly interacting regime in fact provides a venue for disentangling the contributions of both two- 37, 38] and three-body correlations [39 41], and in principle even higher. This sort of direction seems a likely avenue for the study of higher-order Brunnian systems.

Many-body correlations can typically have very nonlocal character, such as in quantum and fractional quantum Hall systems [42]. This is strongly connected to the topological properties of these system which has recently been given a very elegant description in momentum-space through the use of topological invariants akin to the linking number or the genus of a surface [43]. The quantum Spin Hall effect and experimental realizations of so-called topological insulators that have topologically protected conducting surface states are recent highlights of this research [44, 45]. The non-local character of these interesting many-body phases could have some relation to the higher-order Brunnian states that we discuss here.

The non-locality of the Borromean system can be attributed to the defining feature that removing one particle will unbind the system. This implies that no single particle, and no two-particle combination, holds the entire information about the total system structure. This is akin to the many synergetic relationships seen in biological systems in Nature. Taking the Borromean system and generalizing to higher-order Brunnian is a systematic extension of the non-locality. Consider the example of the nine particle with three clusters of three particle second-order Brunnian state discussed previously. Here no single cluster contains the information that the total structure is bound. Furthermore, removing one particle in any one of the clusters will dissociate the clusters, and subsequently the entire structure. This is truly a very non-local behavior similar to the new topological systems intensely studied in condensed-matter and solidstate systems.

A further, yet more speculative implication, is that generalized Brunnian systems might be used to classify excitations in systems that are typically described by conformal field theory such as quantum Hall systems [46]. The correlations in the ground-state or excitations could perhaps be described in terms of generalized Brunnian structures embedded in the many-body environment.

\section{NUCLEAR SYSTEMS}

We start by outlining the Brunnian systems of first order. In three-body Borromean (or Brunnian) systems in nuclei at least two out of the three components must be neutrons, protons or alpha particles in order to maintain the identity of the components in the combined system. Apart from ${ }^{9} \mathrm{Be}$ all established Borromean ground states occur at the proton or neutron dripline and have a core $+2 p$ or core $+2 n$ structure. Systems with several alpha particles are known, but appear as excited states and even the simplest of them, the Hoyle state at 7.65 $\mathrm{MeV}$ in ${ }^{12} \mathrm{C}$, is unbound. Among more complex states, the ground state of ${ }^{123} \mathrm{Xe}$ fulfills the energy requirements for being a Borromean state built of ${ }^{115} \mathrm{Sn}$ and two alpha particles, but it is not clear that a cluster description is appropriate in this case. The core plus two nucleon systems naturally occur at the driplines due to the requirement that the core plus one nucleon system is unbound. In light nuclei where Coulomb barriers for protons are small and the angular momenta are small as well, most Borromean systems (whether in ground states or excited states) appear as two-neutron or two-proton halo nuclei, see [14] that also contains a discussion of the core $+\mathrm{p}+\mathrm{n}$ systems. Two Borromean halo nuclei we shall discuss in the following are ${ }^{6} \mathrm{He}$ and ${ }^{11} \mathrm{Li}$. We mention in passing that there is only one four-body Brunnian system known in nuclei, namely ${ }^{10} \mathrm{C}$ [47].

The Brunnian systems of second order will by definition lie just below the threshold for break-up into three 
three-body Brunnian systems of first order. The energies of these thresholds are easily found and are as follows: Three Hoyle states (we disregards for the moment that it is slightly unbound) would give a second order state close to the 9 alpha threshold in ${ }^{36} \mathrm{Ar}$ at $52.06 \mathrm{MeV}$. The three ${ }^{9} \mathrm{Be}$ threshold is at $48.63 \mathrm{MeV}$ in ${ }^{27} \mathrm{Mg}$, the three ${ }^{6} \mathrm{He}$ threshold is at $27.86 \mathrm{MeV}$ in ${ }^{18} \mathrm{C}$, and the three ${ }^{11} \mathrm{Li}$ threshold lies more than $50 \mathrm{MeV}$ above the unbound ground state resonance in ${ }^{33} \mathrm{~F}$. That the candidate states appear at high excitation energy is natural for cluster states in nuclei as is known e.g. from the Ikeda picture [48, 49] for alpha-cluster states in self-conjugate nuclei.

The candidate states for having a Brunnian structure of second order would have a quite unusual spatial structure and would, as halo nuclei, have a rather large extent. However, being so high up in the continuum implies that there will be plenty of states with which they can mix. They can therefore be expected to behave in close analogy to halo states at high excitation energy that are known [50] to mix with other states rather than to remain unperturbed. In both cases, there is no quantum number or symmetry that preserves the topology or clustering of the structure. The Brunnian structure has to appear "dynamically" and cannot be imposed.

Even without the mixing with other states one may ask whether a Brunnian structure of second order could appear at all. The point is that we in nuclei have only a limited number of building blocks for the Brunnian systems of first order. The interactions between the components of different first order systems can therefore not be neglected and will typically be of the same order as the ones within a given first order system. This will lead to distortions of the first order systems unless the binding energy of the three first order systems in the second order state is extremely low. In other words there is no natural manner of separating the scales to make the appearance of the second order Brunnian structure realistic.

Brunnian states of second order are therefore unlikely to be found in nuclei. Still, related structures may appear and have been considered earlier. Cluster systems in nuclei [49, 51] have been discussed for many years and searches have been made e.g. for states in ${ }^{12} \mathrm{Be}$ with a "two ${ }^{6} \mathrm{He}$ structure". This particular combination may be favoured due to a threshold of only $10.1 \mathrm{MeV}$ which is low compared to other combinations of two first order Brunnian nuclei. Among the other cluster structures discussed are three-center nuclear molecules in heavy carbon isotopes and also non-standard topologies such as Wilkinson's suggestion of a ring of alpha-particles bound by delocalized neutrons [52], but these structures do not involve a Brunnian aspect. For more general topological cluster type many body systems, see [9].

A special case is the addition of neutron pairs to an existing nucleus. The (as yet unreachable) neutron dripline for heavy nuclei is predicted to have many nuclei analogous to ${ }^{8} \mathrm{He}$ and ${ }^{19} \mathrm{~B}$, i.e. where the core $+x \mathrm{n}$ system is unbound for $x=1,3,5, .$. and bound for $x=2,4$ (and even higher). It has even been suggested that this type of structure may appear also for low masses beyond the dripline [53]. However, one would expect the internal structure of the states to rearrange as neutrons are added. For the only case that has been studied in detail today, ${ }^{8} \mathrm{He}$, the structure is much better described by four neutrons around an alpha-particle rather than two di-neutrons around an alpha-particle. Structurally these correspond to Borromean systems with more than three components rather than Brunnian systems.

\section{DISCUSSION AND CONCLUSION}

Mathematical knot theory employs purely topological concepts. This mature discipline was over the years successively generalized to classify and describe geometric structures of any number of rings. About 30 years ago Borromean structure of nuclear clusters was found to exhibit unexpected large spatial extension. This initiated huge research efforts involving large-scale radioactive beam facilities with the aim of localizing and investigating such nuclear structures. This quickly gave rise to the very notion of Borromean systems as bound three-body systems where all three two-body subsystems are unbound. Thus, the Borromean nuclear three-body system was defined in terms of binding arising from interactions bewteen the particles forming the three-body system. The geometric aspect has disappeared from this definition although the basic concept of entangled clusters remains. The same generalization to more particles can then be done, based on binding energies, precisely as for geometric structures.

The definitions can be based on the concept of a link, that is two systems are linked if they are bound or if they can be detached from each other without cutting one of the rings. Correspondingly they are unlinked if they are unbound and hence would fall apart instantaneously. An $(n, k)$ Brunnian system is defined as a system with $n$ particles where all subsystems with $m(\leq k)$ particles are unlinked.

Recently surfaced another generalization to higher order Brunnian systems. We shall confine ourselves to $k=n-1$ and specify the corresponding generalization to the next order for a system of nine particles divided into three clusters forming a Borromean system, where each cluster is a three-body Borromean system. The original topological picture suggests macroscopic systems which easily can be visualized as configurations of a number of entangled rings. It is not unlikely that such structures occur in macroscopic biomolecules consisting of a large number of atoms of molecules. We shall not investigate these possibilities but instead focus on the world of microscopic quantum physics.

We first specify the hamiltonian in general terms to relate to descriptions in microscopic quantum physics. We discuss three subfields of physics where such structures could be found. The interactions in nuclear physics is first of all of short range but modifcations are essentially 
always present due to the long-range repulsive Coulomb interaction. This implies that a bound nuclear structure must have a radius sufficiently small to exploit the short-range attraction. For $s$-waves and neutral particles this is not a strong limitation, but unfortunately all nuclear clusters (except the neutron itself) are charged. The combination of short-range attraction and the repulsive Coulomb force allows two options, that is either be inside or outside the resulting barrier. Being outside means unbound and being inside the barrier implies a small radius. Therefore, a bound system almost inevitably has a relatively small distance between all particles. The necessary cluster configurations $(3 \times 3)$ is then very unlikely in a ground (or low-lying excited) state, simply because the interactions between any pair of particles (within and between three-body subcluster particles) are comparable.

Cold atomic gases are presently investigated in many laboratories with entirely new techniques. It is possible to vary the interaction between pairs of particles by using couplings between excited states depending on externally tunable magnetic fields. By combining with laboratory controlled electromagnetic fields from lasers, both geometries and effective interactions can be varied to a large extent. These systems are therefore tempting candidates for higher order microscopic Brunnian systems. First, three different states must be available becasue all particles in one state, as bosons are allowed to do, would not be able to produce a three-cluster configuration, but rather a condensate. Still, this is possible and achieved, and each state can be prepared to avoid two-body binding while allowing Borromean structure. Then four-body and higher cluster correlations should not be formed with a suitably low density, but the interactions between the three different Borromean systems now also must be of
Borromean nature. This requires experimental investigation and probably a good deal of luck since these interactions cannot be tuned independently.

Another possibility for cold atomic gases is to look for two-dimensional structures where the different Borromean systems should be made in separate planes. The interactions are well-known for polarized molecules in controlled electric fields. It is then possible to apply different fields to tune the interactions between molecules in separate planes. We can then imagine to achieve the desired Borromean conditions both within and between planes.

Condensed matter systems can be of very complex structure. Whether higher order Brunnian systems may be designed or just turn up is difficult to say but far from excluded. However, we have no specific knowledge of structures that suggest themselves in this context, at best only very speculative in connection with non-local interactions in recently studied quantum systems.

One difficulty is that spatially extended clusters are less likely when the number of clusters is larger than three. If several clusters are bound together by shortrange interactions, a barrier is efffectively produced. Its effect is similar (less pronounced) to that of the repulsive long-range Coulomb interaction which either pushes to no binding or to spatially small sizes where the cluster structure is difficult to maintain.

In conclusion, higher order Brunnian systems are, unlikely to occur in nuclei, possible for cold atomic gases either for polarized molecules in two dimensions or populations of three different states in three dimensions, imaginable in condensed matter systems but may be difficult to find or synthesize.
[1] Tait P G 1876 Trans.Roy.Soc.Edin. 28145

[2] Brunn H 1892 Sitz. Bayer. Akad. Wiss. Math-Phys. Klasse, 2277

[3] DeBrunner H 1961 Duke Math. J. 2817

[4] Penney D E 1969 Duke Math. J. 3631

[5] Baas N A 2013 Int. J. Gen. Syst. 42 137; arXiv:1012.2698v2 [cond-mat.quant-gas]

[6] Baas N A and Seeman N C 2012 J. Math. Chem. 50220

[7] Liang C and Mislow K 1994 J.Math.Chem. 1627

[8] Baas N A 2009 Eur. Phys. J. Special Top. 17825

[9] Baas N A 2013 Int. J. Gen. Syst. 42 170; arXiv:1201.6228v1 [math.GM]

[10] Frisch H L and Wassermann E 1961 J.Amer.Chem.Soc. 833789

[11] Mao C, Sun W and Seeman N C 1997 Nature 386137

[12] Zhukov M V, Fedorov D V, Danilin B V, Vaagen J S and Bang J M 1992 Nucl. Phys. A 539177

[13] Ferlaino F and Grimm R 2010 Physics 39

[14] Jensen A S, Riisager K, Fedorov D V and Garrido E 2004 Rev. Mod. Phys. 76215

[15] Lewenstein M et al. 2007 Adv. Phys. 56243

[16] Bloch I, Dalibard J and Zwerger W 2008 Rev. Mod. Phys.
80885

[17] Chin C, Grimm R, Julienne P S and Tiesinga E 2010 Rev. Mod. Phys. 821225

[18] Lahaye T, Menotti C, Santos L, Lewenstein M and Pfau T 2009 Rep. Prog. Phys. 72126401

[19] Carr L D, DeMille D, Krems R V and Ye J 2009 New Jour. Phys. 11055049

[20] Chang M-S, Qin Q, Zhang W, You L and Chapman M S 2005 Nature Phys. 1111

[21] Sadler L E, Higbie J M, Leslie S R, Vengalattore M and Stamper-Kurn D M 2006 Nature 443312

[22] Micheli A, Pupillo G, Büchler H-P and Zoller P 2007 Phys. Rev. A $\mathbf{7 6} 043604$

[23] de Miranda M H Get al. 2011 Nature Phys. 7502

[24] Nielsen E, Fedorov D V and Jensen A S 1997 Phys. Rev. A 563287

[25] Huang S-J et al. 2012 Phys. Rev. A 85055601

[26] Volosniev A G et al. 2013 Preprint arXiv:1312.6535

[27] Armstrong J R, Zinner N T, Fedorov D V and Jensen A S 2010 Europhys. Lett. 9116001

[28] Volosniev A G, Zinner N T, Fedorov D V, Jensen A S and Wunsch B 2011 J. Phys. B 44125301 
[29] Volosniev A G, Fedorov D V, Jensen A S and Zinner N T 2011 Phys. Rev. Lett. 106250401

[30] Ketterle W and Zwierlein M W 2008 Rivista del Nuovo Cimento 31247

[31] Lompe T, Ottenstein T B, Serwane F, Wenz A N, Zürn G and Jochim S 2010 Science 330940

[32] Nakajima S, Horikoshi M, Mukaiyama T, Naidon P and Ueda M 2011 Phys. Rev. Lett. 106143201

[33] Sørensen O, Fedorov D V and Jensen A S Phys. Rev. Lett. 89173002

[34] Dall R G et al. 2011 Nature Comm. 2291

[35] Tan S 2008 Ann. Phys. 323 2952; 323 2971; 3232987

[36] Lee P A, Nagaosa N and Wen X-G 2006 Rev. Mod. Phys. 7817

[37] Stewart J T, Gaebler J P, Drake T E and Jin D S 2010 Phys. Rev. Lett. 104235301

[38] Kuhnle E D, Hu H, Liu X-J, Dyke P, Mark M, Drummond P D, Hannaford P and Vale C J 2010 Phys. Rev. Lett. 105070402

[39] Braaten E, Kang D and Platter L 2011 Phys. Rev. Lett. 106153005
[40] Castin Y and Werner F 2011 Phys. Rev. A 83063614

[41] Wild R J, Makotyn P, Pino J M, Cornell E A and Jin D S 2012 Phys. Rev. Lett. 108145305

[42] Altland A and Simons B 2010 Condensed Matter Field Theory (Cambridge University Press, 2. ed.)

[43] Moore J 2010 Nature 464194

[44] Hasan M Z and Kane C L 2010 Rev. Mod. Phys. 823045

[45] Qi X-L and Zhang S-C 2011 Rev. Mod. Phys. 831057

[46] Ezawa Z F 2008 Quantum Hall Effects (World Scientific 2nd ed.).

[47] Curtis N et al. 2008 Phys. Rev. C 77021301

[48] Ikeda K, Tagikawa N and Horiuchi H 1968 Prog. Theo. Phys. Suppl. Extra 464

[49] von Oertzen W, Freer M and Kanada-En'yo Y 2006 Phys. Rep. 43243

[50] Jensen A S and Riisager K 2000 Phys. Lett. B 48039

[51] Freer M 2007 Rep. Prog. Phys. 702149

[52] Wilkinson D H 1986 Nucl. Phys. A 452296

[53] Jensen A S and Riisager K 1992 Nucl. Phys. A 53745 